\title{
Onco-testicular sperm extraction (onco- TESE) for bilateral testicular tumors: two case reports
}

\author{
Sohgo Tsutsumi ${ }^{1,2}$, Takashi Kawahara ${ }^{1 *}$ D , Teppei Takeshima ${ }^{2}$, Sawako Chiba ${ }^{3}$, Koichi Uemura ${ }^{4}$, Masako Otani ${ }^{3}$, \\ Kota Shimokihara ${ }^{1}$, Yutaro Hayashi ${ }^{1}$, Taku Mochizuki ${ }^{1}$, Daiji Takamoto ${ }^{1}$, Yusuke Hattori ${ }^{1}$, Jun-ichi Teranishi ${ }^{1}$, \\ Yasuhide Miyoshi ${ }^{1}$, Masahiro Yao ${ }^{5}$, Yoshiaki Inayama ${ }^{3}$, Yasushi Yumura ${ }^{1}$ and Hiroji Uemura'
}

\begin{abstract}
Background: Most patients with testicular cancer are infertile; thus, the preservation of the sperm after surgery is an important factor to consider. We report two cases of bilateral testicular cancer in patients who underwent bilateral higher orchiectomy and simultaneous testicular sperm extraction.

Case presentation: Two Asian-Japanese men were referred to our hospital with bilateral testicular tumors. Both of the patients were preoperatively diagnosed with azoospermia and requested testicular sperm extraction at the time of higher orchiectomy. In one patient, sperm was successfully harvested and then frozen. In the other patient, sperm could not be retrieved from the patient's testis. In both patients, the pathological diagnosis was seminoma. Testicular tumors often occur in patients of reproductive age. The preservation of sperm before chemotherapy or bilateral orchiectomy is necessary for patients with testicular tumors who wish to be fathers.
\end{abstract}

Conclusions: Onco-testicular sperm extraction might be an option for patients with testicular cancer and azoospermia or severe oligospermia.

Keywords: Onco-TESE, Azoospermia, Oligospermia, Testicular cancer

\section{Background}

The standard treatment for testicular tumors is higher orchiectomy. Adjuvant chemotherapy and radiation therapy are sometimes added. Most patients with testicular cancer are infertile; thus, preservation of their sperm after surgery is an important factor to consider. We report two cases of bilateral testicular cancer in patients who underwent bilateral higher orchiectomy and simultaneous testicular sperm extraction (onco-TESE). Sperm was successfully harvested from one of these patients.

\section{Case presentations}

\section{Patient 1}

A 38-year-old unmarried Asian-Japanese man with bilateral testicular tumors was referred to our hospital to

\footnotetext{
*Correspondence: takashi_tk2001@yahoo.co.jp; kawahara@yokohama-cu.ac.jp ${ }^{1}$ Department of Urology/Renal Transplantation, Yokohama City University Medical Center, Yokohama, Japan

Full list of author information is available at the end of the article
}

undergo sperm preservation. His right testis was swollen to the size of a chicken's egg; his left testis was normally sized but had a nodule. He had no particular medical history. A laboratory examination showed almost normal results (including lactate dehydrogenase $[\mathrm{LDH}]$ and $\alpha$ fetoprotein $[\mathrm{AFP}])$, with the exception of the human chorionic gonadotropin (HCG) level, which was elevated $(25.8 \mathrm{mIU} / \mathrm{ml})$. The patient's serum testosterone level was within the normal range. An ultrasonographic examination showed a low echoic lesion on the whole right testis and partially on the left testis at the same location as the palpable nodule (Fig. 1a and b). Computed tomography (CT) showed no distant metastasis or lymph node swelling. On the basis of these findings, bilateral testicular tumors (cTxNOM0) were diagnosed, and bilateral orchiectomy was planned. In a sperm examination performed 1 day before surgery, no sperm were detected in $8.2 \mathrm{ml}$ of semen. Owing to the patient's azoospermia, we planned to perform onco-TESE from 


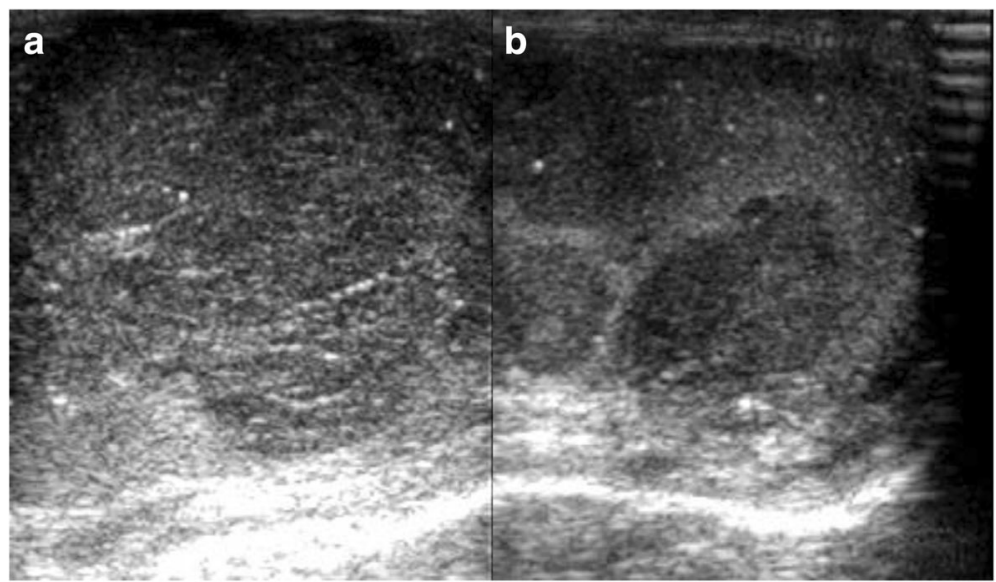

Fig. 1 Ultrasonographic findings in patient 1. a Right testis. b Left testis

his normal left testicular tumor at the time of bilateral orchiectomy. Because the tumorous lesion was easily detected macroscopically, we could retrieve the sperm of his normal left testicular tumor soon after resection. Approximately 1000 sperm were counted and then frozen. The pathological diagnosis was seminoma (pT1N0M0). Most of the right testis was occupied by the tumor. Microscopically, the testicular parenchyma adjacent to the tumor was atrophic, with shrunken seminiferous tubules having thickened basement membranes and diminished numbers of germ cells, a so-called Sertoli cell-only appearance or completely hyalinized. Collections of hyperplastic Leydig cells were also seen. The patient's mean Johnsen score was 1.40 (Figs. 2a and 3a). In the left testis, the parenchyma adjacent to the tumor was also atrophic and showed an almost Sertoli cell-only appearance, but it contained a few seminiferous tubules with sperm (mean Johnsen score 4.38) (Figs. 2b and 3b). The patient's HCG level was normal after surgery, and he has received testosterone supplementation since surgery. He did not receive adjuvant chemotherapy and has had no recurrence in the 0.5 years since surgery.

\section{Patient 2}

A 35-year-old married Asian-Japanese man was referred to our hospital with bilateral swelling of the scrotum. He had no particular medical history. A blood analysis revealed elevated tumor marker levels (LDH $314 \mathrm{IU} / \mathrm{L}$, HCG $27.4 \mathrm{mIU} / \mathrm{ml}$ ). The patient's AFP level and other laboratory data were within the normal limits. CT showed no distant or lymph node metastasis. On the basis of these findings, the patient was preoperatively diagnosed with a bilateral testicular tumor (cTxNOM0). One day prior to bilateral orchiectomy, a semen test was performed, but no sperm were identified (semen volume $1.8 \mathrm{ml}$ ). We planned to perform onco-TESE at the time of surgery because the patient wanted to preserve his sperm. Grossly, a white multinodular tumor occupied the patient's entire left testis, whereas the tumor of the right testis left a compressed area of uninvolved parenchyma (Fig. 4). After the resection of both testes, we examined the normal part of the right testis microscopically, but no sperm were detected. The pathological diagnosis was seminoma (pT1N0M0). Microscopically, the uninvolved parenchyma of the right testis showed

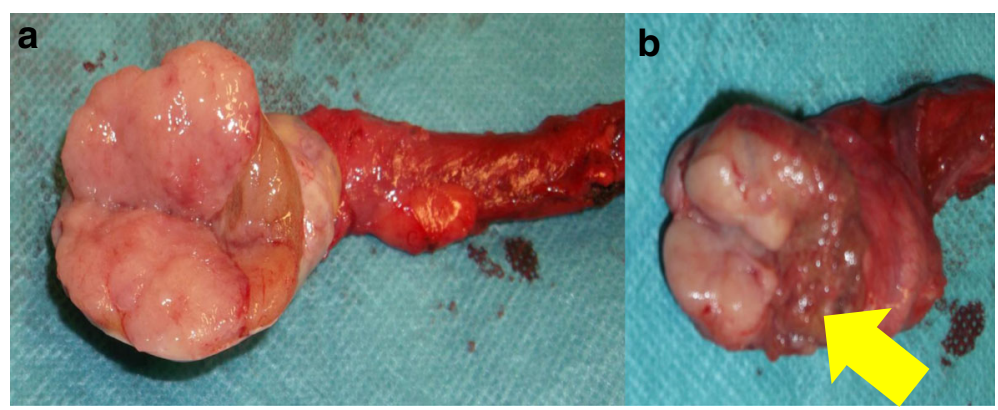

Fig. 2 Macroscopic findings in patient 1. a Right testis. b Left testis (Arrow: normal testis) 


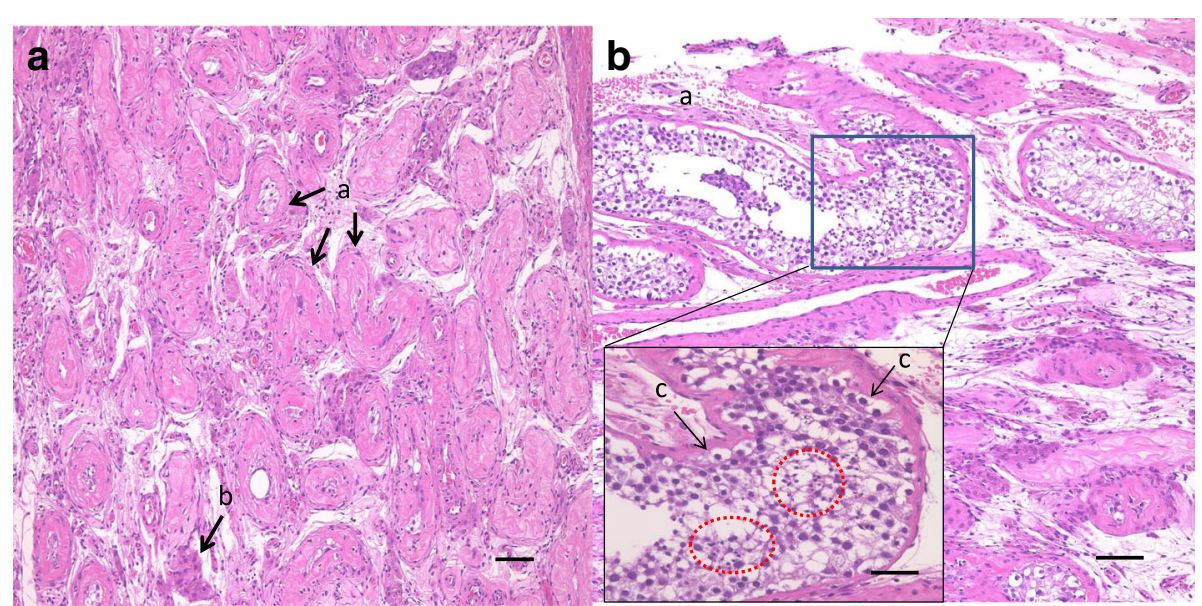

Fig. 3 Histopathological findings using hematoxylin and eosin stain. a Shrunken seminiferous tubules (a) and Leydig cells (b) (bar = $100 \mu \mathrm{m})$. b Seminiferous tubules (a) (bar = $100 \mu \mathrm{m})$; inset: spermatogonia (c); red circles: spermatids (bar $=50 \mu \mathrm{m})$ Mean Johnsen's score count: a 1.40, b 4.38. Arrow: normal tetis

atrophy. In some areas without gross abnormality, tumor cells invaded between preserved seminiferous tubules. Spermatocytes were noted in preserved seminiferous tubules, but spermatogenesis was arrested. The patient's mean Johnsen score was 2.40. The patient's elevated LDH and HCG levels decreased to a normal level after surgery, and no adjuvant therapy was administered. $\mathrm{He}$ received testosterone supplementation after surgery and has shown no recurrence in the 2.5 years since surgery.

\section{Discussion}

Testicular tumors account for $1.0-1.5 \%$ of malignant disease in male patients, and they usually occur in men 15-35 years of age. Bilateral cases account for 1.0-2.0\% of testicular tumors. Heterochronous bilateral testicular tumors are seen four times more frequently than simultaneous bilateral testicular tumors. Seminoma, which is the most common type of bilateral testicular tumor, is seen in $47 \%$ of heterochronous cases and $67 \%$ of simultaneous cases [1]. Although bilateral cases require testosterone replacement therapy, the prognosis is almost the same as that in unilateral cases.

The risk of testicular tumors in male infertility patients is 20 times higher than that in patients who are not infertile. The higher incidence of testicular tumors in infertile patients was thought to be associated with the diminished spermatogenic function of the patients before the development of the tumor. This hypothesis is referred to as testicular dysgenesis syndrome. The syndrome is suggested to be a reason for the development of testicular tumors, a decrease in spermatogenic function, cryptorchidism, and hypospadia [2]. For these reasons, male infertility patients should be screened for testicular tumors.

Onco-TESE is a procedure that allows sperm to be obtained from the normal testis of patients who do not emit sperm prior to cancer therapy. Onco-TESE can be applied to patients with azoospermia, severe

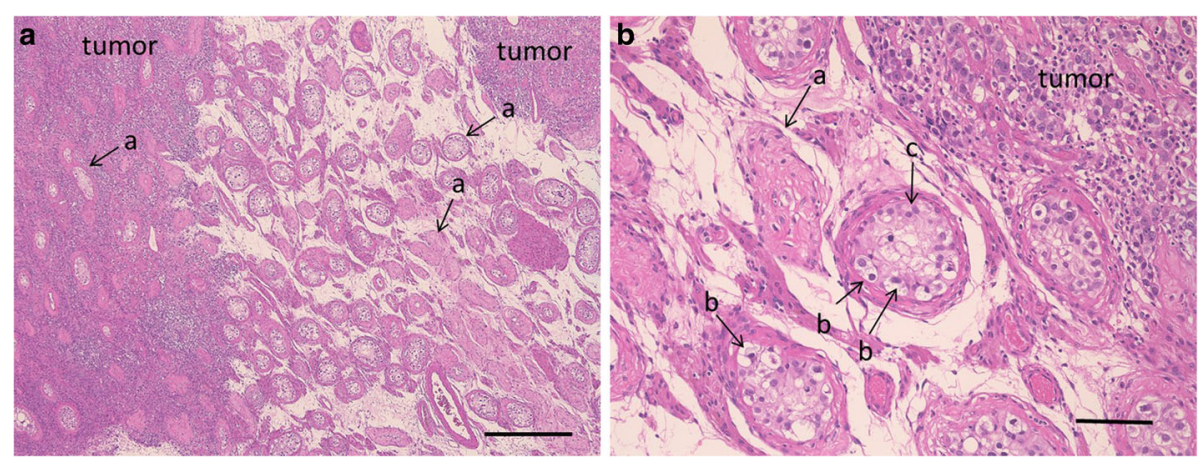

Fig. 4 Histopathological findings using hematoxylin and eosin stain. a Seminiferous tubules (bar $=500 \mu \mathrm{m})$. b Spermatogonia (bar $=100 \mu \mathrm{m})$. c Sertoli cells 
oligospermia, or ejaculation disorder before chemotherapy or in cases involving bilateral testicular tumors. Sperm was frozen for a total of 168 cases at our institute from 2011 to 2016. Fifty-three (31.5\%) of these cases involved patients with testicular tumors. Twenty-six $(60.5 \%)$ of these cases involved patients with bilateral testicular tumors (bilateral, $n=6$; unilateral, $n=20$ ). Five of these patients were azoospermic, and one patient was dysspermic. We performed onco-TESE in three patients. Including our own patients, 43 cases of onco-TESE have been reported in the literature [3-6]. In 22 (51.2\%) cases, sperm was successfully preserved, including 14 patients $(53.8 \%)$ with testicular tumors. The previous reports noted that pregnancy was achieved in four cases using sperm extracted via onco-TESE, with a healthy child delivered in three of the four cases. A correlation between tumor size and spermatogenesis in patients with testicular tumors has been reported. Jeremy et al. reported that spermatogenesis was observed in $86 \%$, $81 \%$, and $57 \%$ of cases involving tumors of $1 \mathrm{~cm}, 2 \mathrm{~cm}$, and $5 \mathrm{~cm}$ in size, respectively [7].

Although onco-TESE is useful, the number of institutes in which surgeons perform the procedure is limited. When these restrictions are cleared, onco-TESE will become an important option for preserving fertility. Regarding infertility, CT might have some effects. But, based on cancer control, CT examination should be recommended.

\section{Conclusions}

We describe two cases in which onco-TESE and resection were performed simultaneously in patients with bilateral testicular tumors.

\section{Acknowledgements}

Kimitou Kobayashi and Mizuki Yamamoto who are embryologists in

Yokohama City University Medical Center, supported this study.

\section{Funding}

Grants were received from the Grants-in-Aid for Scientific Research Program (KAKENHI grant 16 K20152), from the Ministry of Education, Culture, Sports, Science and Technology of Japan, and from the 2016-2017 Research Development Fund (WJ2810) of Yokohama City University.

\section{Availability of data and materials}

Owing to ethical restrictions, the raw data on which this paper is based are available upon request from the corresponding author.

\section{Authors' contributions}

ST, TK, and YY conceived of and designed the experiments. ST, TK, YY, SC, and $\mathrm{MO}$ analyzed data. ST, TT, KU, KS, YH, TM, DT, YH, JT, YM, MY, YI, YY, and $\mathrm{HU}$ performed the experiments. ST, TK, SC, MO, and YY wrote the paper. All authors read and approved the final manuscript.

\section{Competing interests}

The authors declare that they have no competing interests.

\section{Consent for publication}

Written informed consent was obtained from the patients for publication of this case report and any accompanying images. Copies of the written consents are available for review by the Editor-in-Chief of this journal.
Ethics approval and consent to participate

The present study was approved by the institutional review board of Yokohama City University Medical Center, and written informed consent was obtained from the patients.

\section{Publisher's Note}

Springer Nature remains neutral with regard to jurisdictional claims in published maps and institutional affiliations.

\section{Author details}

${ }^{1}$ Department of Urology/Renal Transplantation, Yokohama City University Medical Center, Yokohama, Japan. ${ }^{2}$ Reproduction Center, Department of Urology, Yokohama City University Medical Center, Yokohama, Japan. ${ }^{3}$ Division of Diagnostic Pathology, Yokohama City University Medical Center, Yokohama, Japan. ${ }^{4}$ Department of Urology, Hiratsuka Kyosai Hospital, Hiratsuka, Japan. ${ }^{5}$ Department of Urology, Yokohama City University Graduate School of Medicine, Yokohama, Japan.

Received: 17 February 2017 Accepted: 28 April 2017

Published online: 17 May 2017

\section{References}

1. Klatte T, de Martino M, Arensmeier K, Reiher F, Allhoff EP, Klatte D. Management and outcome of bilateral testicular germ cell tumors: a 25-year single center experience. Int J Urol. 2008;15(9):821-6.

2. Skakkebaek NE, Rajpert-De Meyts E, Main KM. Testicular dysgenesis syndrome: an increasingly common developmental disorder with environmental aspects. Hum Reprod. 2001;16(5):972-8.

3. Roque M, Sampaio M, Salles PG, Geber S. Onco-testicular sperm extraction: birth of a healthy baby after fertility preservation in synchronous bilateral testicular cancer and azoospermia. Andrologia. 2015:47(4):482-5.

4. Furuhashi K, Ishikawa T, Hashimoto H, Yamada S, Ogata S, Mizusawa Y, et al. Onco-testicular sperm extraction: testicular sperm extraction in azoospermic and very severely oligozoospermic cancer patients. Andrologia. 2013;45(2): 107-10

5. Schrader M, Müller M, Sofikitis N, Straub B, Krause H, Miller K. "Onco-tese": testicular sperm extraction in azoospermic cancer patients before chemotherapy—new guidelines? Urology. 2003;61 (2):421-5.

6. Descombe L, Chauleur C, Gentil-Perret A, Aknin-Seifer I, Tostain J, Lévy R. Testicular sperm extraction in a single cancerous testicle in patients with azoospermia: a case report. Fertil Steril. 2008;90(2):443.e1-4.

7. Choy JT, Wiser HJ, Bell SW, Cashy J, Brannigan RE, Kohler TS. Predictors of spermatogenesis in orchiectomy specimens. Urology. 2013;81(2):288-92.

Submit your next manuscript to BioMed Central and we will help you at every step:

- We accept pre-submission inquiries

- Our selector tool helps you to find the most relevant journal

- We provide round the clock customer support

- Convenient online submission

- Thorough peer review

- Inclusion in PubMed and all major indexing services

- Maximum visibility for your research

Submit your manuscript at www.biomedcentral.com/submit
C Biomed Central 